01

\title{
Фазовые превращения в однокомпонентных многофазных системах: фазово-полевой подход
}

\author{
() В.Г. Лебедев
}

Научный центр металлургической физики и материаловедения УдмФИЦ УрО РАН, 426067 Ижевск, Россия

e-mail: Ivg@udsu.ru

Поступило в Редакцию 12.07.2021 г.

В окончательной редакции 8 ноября 2021 г.

Принято к публикации 12 ноября 2021 г.

Рассмотрены проблемы построения многофазной модели фазового поля для процессов фазовых переходов первого рода. Исходя из энергии Гиббса полной системы, выраженной через антисимметризованные комбинации фазовых полей, показано, что из условия ее монотонного убывания следуют уравнения диссипативной динамики локально-неравновесной системы, сохраняющие нормировку суммы переменных на единицу и наследующие свойства ранее известной двухфазной модели.

Ключевые слова: многофазные системы, фазовые превращения, локально-неравновесные процессы, диссипативные системы со связями.

DOI: $10.21883 / J T F .2022 .02 .52007 .215-21$

\section{Введение}

Моделирование процессов формирования микроструктур при фазовых переходах имеет важное значение для разработки новых технологий [1]. К наиболее эффективным и достоверным подходам в моделировании микроструктур относится метод фазового поля [2]. Наиболее исследованы двухфазные модели [3-6], позволяющие описывать взаимодействие зерен или рост дендритных структур в материалах. Для таких моделей пройден путь от феноменологических идей диффузной границы [7-11] до термодинамически согласованных [12-14] моделей. Ситуация в многофазных системах [15-21] более сложная, поскольку зарождение новых фаз на границе двух фаз не сводится к уравнениям [3-6] или [12-14]. Основная проблема состоит в наличии голономной связи [22] для параметров порядка фаз (называемых фазовыми полями).

Действительно, в изотропных материалах динамика системы с $N$ фазами описывается вектором с $N$ компонентами $\varphi_{\alpha}(\alpha=1 \ldots N)$, соответствующими каждой из фаз. Фазовое поле $\varphi_{\alpha}$ однозначно характеризует (за исключением диффузной границы между фазами) наличие фазы $\alpha$ в данной точке пространства-времени. Для равновесной фазы примем значение $\varphi_{\alpha}=1$, а внутри остальных фаз $\varphi_{\alpha}=0$. Поперек диффузной границы фазовое поле меняется быстро, но непрерывно, предполагая выполние связи

$$
\sum_{\alpha=1}^{N} \varphi_{\alpha}=1,
$$

которая не позволяет считать $\varphi_{\alpha}$ независимыми переменными. Анизотропия, характерная для кристаллических фаз, приводит к дополнительным степеням свободы [21] (фазовым полям, контролирующим направление роста) со своей связью вида (1). Явное разрешение связи (1) за счет выделения зависимой фазы не удовлетворительно ни с термодинамической точки зрения, ни с прагматической позиции численного моделирования. Неявное использование связей в вариационных задачах известно достаточно давно. Универсальными подходами в таких задачах являются методы множителей Лагранжа [23] и штрафных функций [24]. Но применение в релаксационной динамике диссипативных систем на сегодня совершенно не обосновано. Тем не менее множители Лагранжа [23] активно использовались в работах [15-21].

Другой подход, связанный с выбором независимых неизбыточных переменных и уравнений динамики, удовлетворяющих связи (1), был предложен еще в работе [16]. Несмотря на достижения [15-21], используемые на данный момент многофазные модели [10,11], являясь развитием модели [16], не сохраняют преемственности с описанием двухфазных систем [2-5], в частности в них не выполнены условия термодинамической устойчивости фаз (4) по Кесслеру [25], вследствие чего равновесное состояние в отличие от двухфазных моделей является квазипериодической функцией. Поэтому, несмотря на обилие выполненных расчетов, задача согласованного описания фазовых превращений в многофазных системах до сих пор далека от завершения.

Цель настоящей работы - получить модель фазового поля для фазовых превращений в многокомпонентных систем в неизбыточных переменных. Представленная модель согласована с ранее известными двухфазными моделями, например [26]. Для согласования использован принцип парности взаимодействия фаз. Принцип парности предполагает, что многофазная модель на границе фаз должна вырождаться до взаимодействия каждой из возможных пар фаз, в то же время не допуская зарожде- 
ния новых. Поскольку динамика фазовых превращений в однокомпонентных системах определяется исключительно разностью потенциалов Гиббса, что является более простым случаем по сравнению с растворами, однокомпонентная система использована для более четкого понимания того, как реализовать такой подход. Исходя из переменных Штайнбаха [16] в изотропной модели фазового поля и требования монотонного убывания потенциала Гиббса в процессе релакции, получена модель, обладающая необходимым поведением. Последнее проверяется как аналитически, так и численно, на основе моделирования затвердевания расплава гафния (Hf), приводящего к конкурентному росту двух твердых фаз из расплава.

\section{1. Многофазное описание на основе принципа парности}

Переформулируем многофазную модель [10,11] в независимых переменных антисимметричных комбинаций фазовых полей [16], сохраняя общую структуру двухфазной модели [26]. Для вывода потребуем монотонного убывания полного потенциала Гиббса всей системы [27], достигающего минимума в состоянии равновесия. Для этого выразим его через антисимметричные переменные. Рассмотрим различные физические вклады в энергию Гиббса многофазной однокомпонентной системы.

\section{1. Вклад поверхности}

В случае многофазных систем из-за избыточности переменных $\varphi_{\alpha}$ в качестве независимых величин выберем антисимметричные комбинации [16]

$$
\psi_{\alpha \beta}=\varphi_{\alpha}-\varphi_{\beta} .
$$

Плотность диффузной границы запишем как сумму по независимым парам индексов $(\alpha, \beta)$ :

$$
\varepsilon_{k}=\frac{1}{8} \sum_{\alpha, \beta=1}^{N} \sigma_{\alpha \beta}\left(\nabla \psi_{\alpha \beta}\right)^{2},
$$

где $\sigma_{\alpha \beta}$ связано с поверхностной энергией границы между фазами $(\alpha, \beta)$. Множитель $1 / 8$ появляется в выражении (2) из-за степени и симметрии $\sigma_{\alpha \beta}=\sigma_{\beta \alpha}$.

\section{2. Потенциальный барьер между фазами}

Следуя Штайнбаху [16], заменим хорошо известный double-well-потенциал, см. [26]:

$$
\varepsilon_{p}=\frac{1}{2} W g(\varphi) \equiv \frac{1}{2} W \varphi^{2}(1-\varphi)^{2}
$$

на выпуклый полином более низкого порядка, также выразив его через антисимметричную комбинацию. Формальное обобщение на многофазный случай дает

$$
\varepsilon_{p}=\frac{1}{2} \sum_{\alpha, \beta=1}^{N} W_{\alpha \beta} \psi_{\alpha \beta}^{2},
$$

где $W_{\alpha \beta}$ как и прежде определяет величину энергетического барьера между фазами $(\alpha, \beta)$.

\section{3. Равновесный термодинамический вклад}

Представим равновесный термодинамический вклад многофазной системы однокомпонентной системы [26]:

$$
\varepsilon_{e}=\sum_{\alpha=1}^{N} \varphi_{\alpha} G_{\alpha}(T)
$$

через комбинации

$$
\varepsilon_{e}=\sum_{\alpha, \beta=1}^{N} \psi_{\alpha \beta} \Delta G^{\alpha \beta},
$$

где $\Delta G^{\alpha \beta}=G^{\alpha}(T)-G^{\beta}(T)$.

\section{4. Локально-неравновесные процессы}

Для описания быстро протекающих фазовых превращений к объемной плотности энергии Гиббса добавим неравновесный кинетический вклад

$$
\varepsilon_{k}=\frac{1}{2} \Gamma \sum_{\alpha=1}^{N} \dot{\varphi}_{\alpha}^{2},
$$

учитывающий сокращение объема фазового пространства системы в неравновесных процессах [27]. Параметр Г подлежит определению в дальнейшем.

\section{5. Производная энергии Гиббса по времени}

Собирая вместе плотности энергий (2), (4), (6), (7), получаем полную энергию многофазной системы

$$
\begin{aligned}
G(t)= & \int \sum_{\alpha, \beta=1}^{N}\left[\frac{1}{2} \Gamma \dot{\varphi}_{\alpha} \dot{\varphi}_{\beta} \delta_{\alpha \beta}+\frac{1}{8} \sigma_{\alpha \beta}\left(\nabla \psi_{\alpha \beta}\right)^{2}\right. \\
& \left.-\frac{1}{2} W_{\alpha \beta} \psi_{\alpha \beta}^{2}+3 \psi_{\alpha \beta} \Delta G^{\alpha \beta}\right] d \Omega .
\end{aligned}
$$

Соответствующая производная по времени равна

$$
\begin{aligned}
\frac{d G(t)}{d t}= & \int \sum_{\alpha=1}^{N} \dot{\varphi}_{\alpha} \sum_{\beta=1}^{N}\left[\Gamma \ddot{\varphi}_{\beta} \delta_{\alpha \beta}-\frac{1}{2} \sigma_{\alpha \beta} \nabla^{2} \psi_{\alpha \beta}\right. \\
& \left.-2 W_{\alpha \beta} \psi_{\alpha \beta}+6 \Delta G^{\alpha \beta}\right] d \Omega \leq 0
\end{aligned}
$$

и формально учитывает вклад в изменение $\alpha$-й фазы за счет всех возможных фаз системы. Однако в реальности число сосуществующих фаз вблизи равновесия должно быть ограничено из-за минимизации поверхностной энергии. Исходя из этого, используем принцип парности взаимодействия фаз. 


\section{6. Принцип парности}

Под этим принципом будем понимать условие, что $d G(t) / d t \leq 0$ должно быть выполнено только с учетом тех фаз, которые непосредственно соприкасаются друг с другом внутри диффузной границы. Иными словами, в выражении (9) вставим квадрат проекционного оператора $\hat{P}_{\alpha} \hat{P}_{\beta} \geq 0$ учитывающего, что внутри диффузной границы имеются обе фазы $\alpha$ и $\beta$

$$
\begin{aligned}
\frac{d G(t)}{d t}= & \int \sum_{\alpha=1}^{N} \dot{\varphi}_{\alpha} \sum_{\beta=1}^{N} \hat{P}_{\alpha} \hat{P}_{\beta}\left[\Gamma \ddot{\varphi}_{\beta} \delta_{\alpha \beta}-\frac{1}{2} \sigma_{\alpha \beta} \nabla^{2} \psi_{\alpha \beta}\right. \\
& \left.-2 W_{\alpha \beta} \psi_{\alpha \beta}+6 \Delta G^{\alpha \beta}\right] d \Omega \leq 0 .
\end{aligned}
$$

Конкретный выбор проекционных операторов в каждом из слагаемых не однозначен, но должен сохранять знакоопределенность выражения (10). Сравнение с двухфазной задачей позволяет принять

$$
\hat{P}_{\alpha} \hat{P}_{\beta}=\varphi_{\alpha} \varphi_{\beta}
$$

для вкладов потенциальных барьеров и термодинамических потенциалов. В то же время, чтобы сохранить в наиболее простом вид границы, для кинетического вклада ограничимся произведением функций Хэвисайда:

$$
\hat{P}_{\alpha} \hat{P}_{\beta}=\theta\left(\left|\nabla \varphi_{\alpha}(x)\right|\right) \theta\left(\left|\nabla \varphi_{\beta}(x)\right|\right),
$$

где $\theta(a)=1$ при $a \geq 0$ и $\theta(a)=0$ при $a<0$. Полагая

$$
\theta_{\alpha} \equiv \theta\left(\left|\nabla \varphi_{\alpha}(x)\right|\right),
$$

перепишем (10) в следующем виде:

$$
\begin{aligned}
\frac{d G(t)}{d t}= & \int \sum_{\alpha} \dot{\varphi}_{\alpha} \sum_{\beta}\left[\Gamma \ddot{\varphi}_{\beta} \delta_{\alpha \beta}-\frac{1}{2} \sigma_{\alpha \beta} \theta_{\alpha} \theta_{\beta} \nabla^{2} \psi_{\alpha \beta}\right. \\
& \left.-2 W_{\alpha \beta} \varphi_{\alpha} \varphi_{\beta} \psi_{\alpha \beta}+6 \varphi_{\alpha} \varphi_{\beta} \Delta G^{\alpha \beta}\right] d \Omega \leq 0,
\end{aligned}
$$

откуда следуют уравнения фазового поля многофазной модели:

$$
\begin{gathered}
\tau \ddot{\varphi}_{\alpha}+\dot{\varphi}_{\alpha}=M_{0} \sum_{\beta \neq \alpha}\left[\frac{1}{2} \sigma_{\alpha \beta} \theta_{\alpha} \theta_{\beta} \nabla^{2}\left(\varphi_{\alpha}-\varphi_{\beta}\right)\right. \\
\left.-2 W_{\alpha \beta} \varphi_{\alpha} \varphi_{\beta}\left(\varphi_{\beta}-\varphi_{\alpha}\right)-6 \varphi_{\alpha} \varphi_{\beta} \Delta G^{\alpha \beta}\right]
\end{gathered}
$$

где $\tau=\Gamma M_{0}-$ характерное время релаксации. Величина $M_{0}-$ мобильность фазового поля, обычно являющаяся функцией температуры. $M_{0}$ определяется из экспериментов по кинетике затвердевания [29], в то время как для определения остальных параметров достаточно условий равновесия [26].

Нетрудно видеть, что сумма уравнений (14) тождественна равна нулю, поэтому если в начальный момент сумма фаз равна единице в соответствии с (1), то она останется таковой и в последующие моменты времени. Отметим, что это возможно только при условии равных мобильностей $M_{0}$ и равных времен релаксации $\tau$ для всех фаз.

В предельном случае, когда контактируют только две фазы, условно $\varphi_{1}$ и $\varphi_{2}$ имеем

$$
W_{12}=W_{21}=W_{0}, \sigma_{12}=\sigma_{21}=\sigma_{0}, \varphi_{1}=\varphi, \varphi_{2}=1-\varphi .
$$

В этом случае получаем два уравнения

$$
\begin{aligned}
\tau \ddot{\varphi}_{1}+\dot{\varphi}_{1}= & \tau \ddot{\varphi}+\dot{\varphi}=M_{0}\left[\sigma_{0} \nabla^{2} \varphi-2 W_{0} \varphi(1-\varphi)(1-2 \varphi)\right. \\
& \left.-6 \varphi(1-\varphi) \Delta G^{(12)}\right], \\
\tau \ddot{\varphi}_{2}+\dot{\varphi}_{2}= & -(\tau \ddot{\varphi}+\dot{\varphi})=M_{0}\left[-\sigma_{0} \nabla^{2} \varphi+2 W_{0} \varphi(1-\varphi)\right. \\
& \left.\times(1-2 \varphi)-6 \varphi(1-\varphi) \Delta G^{(21)}\right],
\end{aligned}
$$

которые совпадают с точностью до знака.

\section{2. Потенциалы Гиббса}

Рассмотрим динамику формирования фаз в чистом гафнии. У гафния имеются три устойчивых (при разных температурах) фазы и его поведение позволяет продемонстрировать возможности многофазной модели. Аналитические выражения для потенциалов Гиббса как функций температуры взяты из работы [28]. Графики потенциалов Гиббса наиболее низкоэнергетических потенциалов Гиббса приведены на рис. 1. Из них видно, что в расплаве, при понижении температуры до $T=2000 \mathrm{~K}$, кроме жидкой фазы, могут возникать (метастабильная) фаза HCP_A3 и устойчивая фаза BCC_A2.

\section{3. Численный алгоритм}

Поскольку основная цель настоящей работы состоит в выводе уравнений, описывающих процессы фазовых

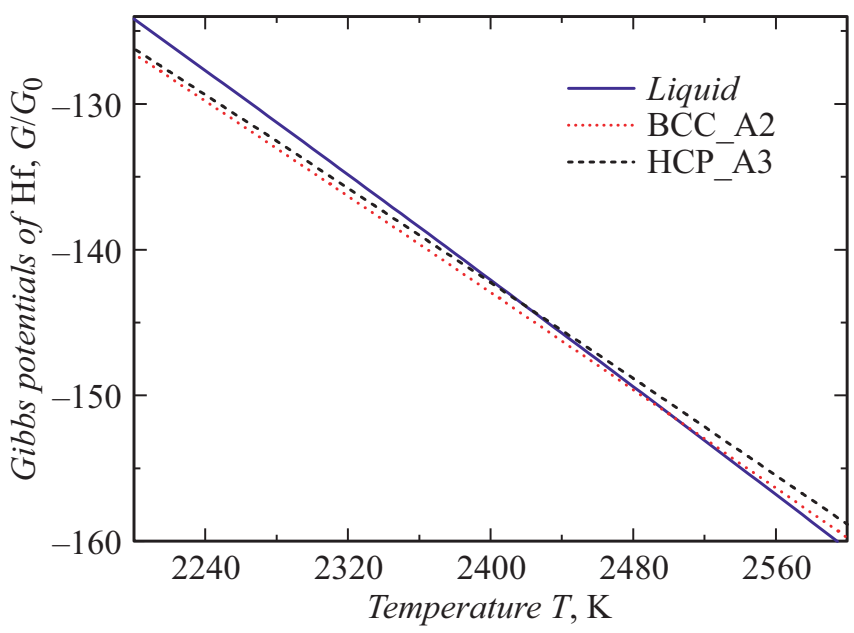

Pис. 1. Мольные потенциалы Гиббса фаз Liquid, BCC_A2, НCР_А3 для чистого гафния [28]. 
превращений в чистых (однокомпонентных) многофазных системах (14), в работе не преследуется задача сопоставления с экспериментом. Представленные далее результаты численных расчетов тоже в основном преследуют иллюстративную цель: показать, что при численном решении уравнений (14) соотношение (1) всегда будет выполнено в процессе структурообразования.

Расписывая уравнения (14) для трех фаз, имеем систему уравнений

$$
\begin{aligned}
\tau \ddot{\varphi}_{1}+\dot{\varphi}_{1}= & M_{0}\left[\sigma_{12} \vartheta_{1} \vartheta_{2} \nabla^{2}\left(\varphi_{1}-\varphi_{2}\right)+\sigma_{13} \vartheta_{1} \vartheta_{3} \nabla^{2}\left(\varphi_{1}-\varphi_{3}\right)\right. \\
& -2 \varphi_{1}\left(W_{12} \varphi_{2}\left(\varphi_{1}-\varphi_{2}\right)+W_{13} \varphi_{3}\left(\varphi_{1}-\varphi_{3}\right)\right) \\
& \left.-6 \varphi_{1}\left(\varphi_{2} \Delta G^{(12)}+\varphi_{3} \Delta G^{(13)}\right)\right] \\
\tau \ddot{\varphi}_{2}+\dot{\varphi}_{2}= & M_{0}\left[\sigma_{21} \vartheta_{2} \vartheta_{1} \nabla^{2}\left(\varphi_{2}-\varphi_{1}\right)+\sigma_{23} \vartheta_{2} \vartheta_{3} \nabla^{2}\left(\varphi_{2}-\varphi_{3}\right)\right. \\
& -2 \varphi_{2}\left(W_{21} \varphi_{1}\left(\varphi_{2}-\varphi_{1}\right)+W_{23} \varphi_{3}\left(\varphi_{2}-\varphi_{3}\right)\right) \\
& \left.-6 \varphi_{2}\left(\varphi_{1} \Delta G^{(21)}+\varphi_{3} \Delta G^{(23)}\right)\right] \\
\tau \ddot{\varphi}_{3}+\dot{\varphi}_{3}= & M_{0}\left[\sigma_{31} \vartheta_{3} \vartheta_{1} \nabla^{2}\left(\varphi_{3}-\varphi_{1}\right)+\sigma_{32} \vartheta_{3} \vartheta_{2} \nabla^{2}\left(\varphi_{3}-\varphi_{2}\right)\right. \\
& -2 \varphi_{3}\left(W_{31} \varphi_{1}\left(\varphi_{3}-\varphi_{1}\right)+W_{32} \varphi_{2}\left(\varphi_{3}-\varphi_{2}\right)\right) \\
& \left.-6 \varphi_{3}\left(\varphi_{1} \Delta G^{(31)}+\varphi_{2} \Delta G^{(32)}\right)\right]
\end{aligned}
$$

Для упрощения численного расчета далее будем считать, что локальная неравновесность отсутствует: $\tau=0$, а величины барьеров и поверхностная энергия на всех границах одинаковы:

$$
\begin{gathered}
W_{12}=W_{13}=W_{23}=W_{0}, \\
\sigma_{12}=\sigma_{13}=\sigma_{23}=\sigma_{0} .
\end{gathered}
$$

Выбирая масштабы времени и пространства, после перехода к безразмерным переменным имеем

$$
\begin{gathered}
\dot{\varphi}_{1}=\vartheta_{1} \vartheta_{2} \nabla^{2}\left(\varphi_{1}-\varphi_{2}\right)+\vartheta_{1} \vartheta_{3} \nabla^{2}\left(\varphi_{1}-\varphi_{3}\right) \\
-2 \varphi_{1}\left(\varphi_{2}\left(\varphi_{1}-\varphi_{2}\right)+\varphi_{3}\left(\varphi_{1}-\varphi_{3}\right)\right) \\
-6 \varphi_{1}\left(\varphi_{2} \Delta \bar{G}^{(12)}+\varphi_{3} \Delta \bar{G}^{(13)}\right), \\
\dot{\varphi}_{2}=\vartheta_{2} \vartheta_{1} \nabla^{2}\left(\varphi_{2}-\varphi_{1}\right)+\vartheta_{2} \vartheta_{3} \nabla^{2}\left(\varphi_{2}-\varphi_{3}\right) \\
-2 \varphi_{2}\left(\varphi_{1}\left(\varphi_{2}-\varphi_{1}\right)+\varphi_{3}\left(\varphi_{2}-\varphi_{3}\right)\right) \\
-6 \varphi_{2}\left(\varphi_{1} \Delta \bar{G}^{(21)}+\varphi_{3} \Delta \bar{G}^{(23)}\right), \\
\dot{\varphi}_{3}=\vartheta_{3} \vartheta_{1} \nabla^{2}\left(\varphi_{3}-\varphi_{1}\right)+\vartheta_{3} \vartheta_{2} \nabla^{2}\left(\varphi_{3}-\varphi_{2}\right) \\
-2 \varphi_{3}\left(W_{31} \varphi_{1}\left(\varphi_{3}-\varphi_{1}\right)+W_{32} \varphi_{2}\left(\varphi_{3}-\varphi_{2}\right)\right) \\
-6 \varphi_{3}\left(\varphi_{1} \Delta \bar{G}^{(31)}+\varphi_{2} \Delta \bar{G}^{(32)}\right),
\end{gathered}
$$

где $\Delta \bar{G}^{(\alpha \beta)}$ - безразмерные разности потенциалов Гиббса. Последние уравнения в векторном виде запишем как

$$
\dot{\varphi}=L(\varphi),
$$

для моделирования которых будем использовать метод конечных разностей. Для явной схемы имеем $L$ :

$$
\varphi^{(n+1)}=\varphi^{(n)}+\Delta t L\left(\varphi^{(n)}\right) .
$$

Из-за нелинейности правой части задачи (19) расчет заведомо должен выполняться с малым шагом по времени, поэтому в настоящей работе не рассматривались неявные методы и соответствующие условия устойчивости. Устойчивость явной схемы (19) контролировалась эмпирически выбором шага. Для одномерной постановки явная схема устойчива на решетке размером $N \approx 1000-2000$ при $\Delta t \leq 0.325 \Delta x^{2}$. Для двумерной постановки (на решетке $N \approx 500 \times 500)$ - решение устойчиво при $\Delta t \leq 0.145 \Delta x^{2}$ где $\Delta x-$ пространственный шаг.

\section{4. Задача направленного затвердевания}

Рассмотрим динамику формирования фаз в гафнии для задачи направленного затвердевания. В качестве начального условия в задаче направленного затвердевания использована комбинация фаз Liquid и HCP_A3 (рис. 2,a).

Первоначально заданные фазы не находятся в равновесии, поэтому межфазная граница проходит в движение. При движении границы раздела фаз Liquid-HCP_A3 (рис. $2, b$ ) возникает неустойчивость, которая приводит к искажению профиля жидкой фазы и возникновению „зародыша“ фазы ВCC_A2. На рис. 2, $b$ - появление новой фазы. Далее (рис. 3,a) показано формирование полноценной устойчивой фазы BCC_A2 (фазовое поле для фазы BCC_A2 достигает значения единицы), внутри объема которой фазы Liquid, HCP_A3 постепенно перестают соприкасаться друг с другом. На рис. 3, $b$ представлено вытеснение метастабильных фаз устойчивой фазы BCC_A2. Из-за того, что разность потенциалов Гиббса между жидкой и устойчивой твердой фазой выше, чем между твердыми фазами, скорость движения границы существенно превышает скорость движения межфазной границы для твердых фаз.

Поскольку вычисление правой части выражения (29) проводилось по известным значениям $\varphi^{(n)}$, для которых условие (1) выполнено, это условие тожественно выполнялось на следующем шаге по времени. Построение неявных методов решения уравнений многофазной системы пока остается актуальной нерешенной задачей.

\section{5. Двумерное моделирование}

Моделирование выполнено на квадратной решетке размером $N \approx 500 \times 500$. Рассматривается изотропная 


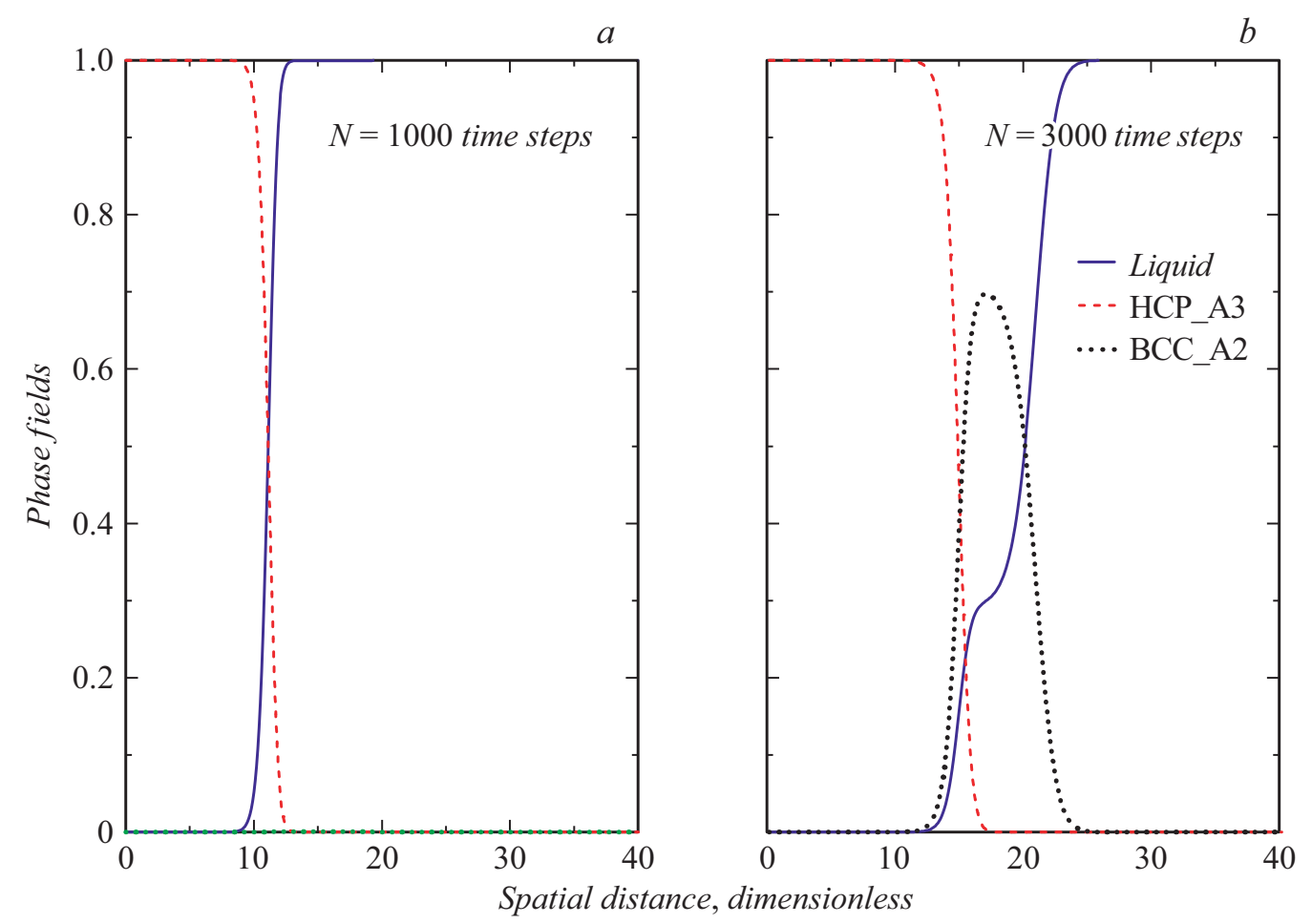

Рис. 2. Процесс появления фазы ВСС_А2 на границе между фазами Liquid и HCP_A3 для чистого гафния при $T=2000$ K.

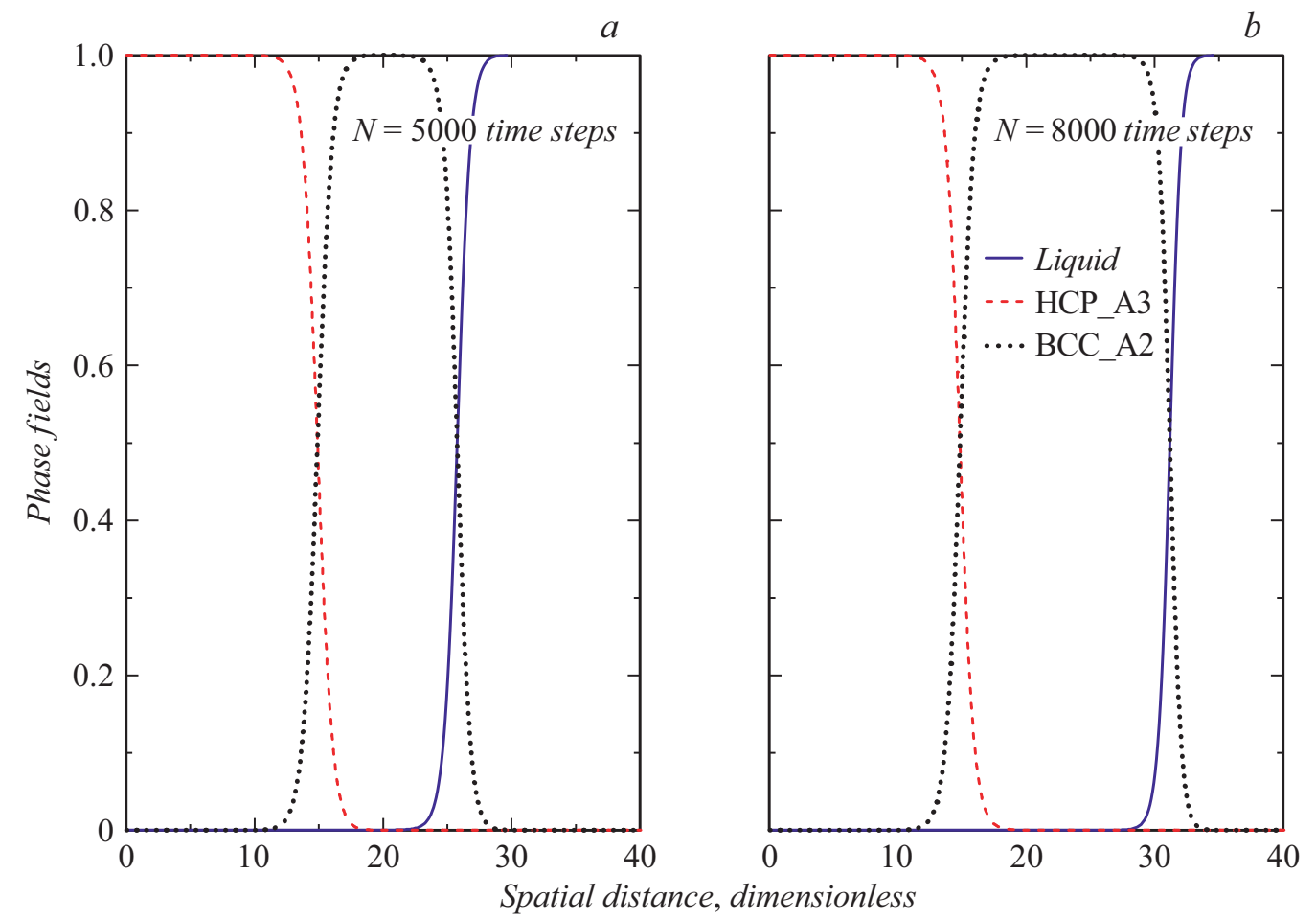

Рис. 3. Формирование устойчивой фазы ВСС_А2 на границе метастабильных фаз для чистого гафния при $T=2000 \mathrm{~K}$ (эволюция слева направо).

модель. Начальное состояние соответствует (псевдо) случайному расположению сферических зародышей фазы НCP_A3 (фазовое поле которой изображено крас- ным (в онлайн версии)) внутри жидкой фазы гафния (фазовое поле отмечено синим (в онлайн версии)) при $T=2000 \mathrm{~K}$ (рис. 4). Обе перечисленные фазы метаста- 
бильны при данной температуре, поэтому в окрестности каждого сферического зародыша заданы флуктуации значения фазового поля порядка $10^{-8}$, соответствующего фазе BCC_A2.

Медленное движение границы фазы BCC_A2 внутри жидкой фазы провоцирует появление неустойчивости и рост фазового поля ВCС_A2 (отмеченного зеленым цветом (в онлайн версии)) на границе между фазами

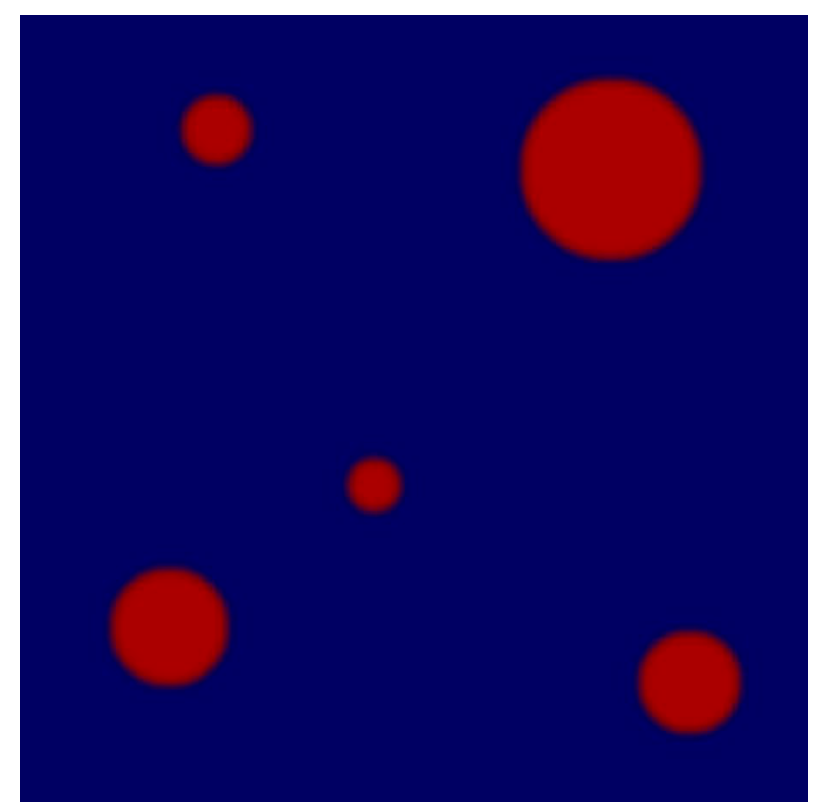

Рис. 4. Начальное состояние: зародыши НСР_А3 фазы (красные круги (в онлайн версии)) внутри фазы Liquid (синий фон (в онлайн версии)) для гафния при $T=2000 \mathrm{~K}$.

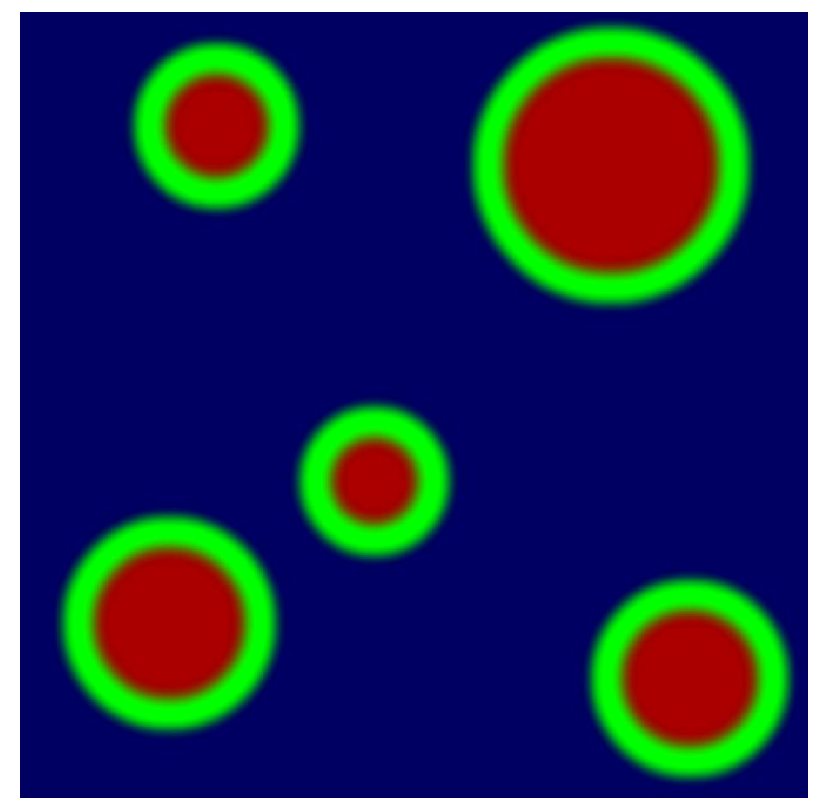

Рис. 5. Появление фазы ВСС_A2 (зеленый (в онлайн версии)) на границе между фазами Liquid и HCP_A3 для гафния при $T=2000 \mathrm{~K}$.

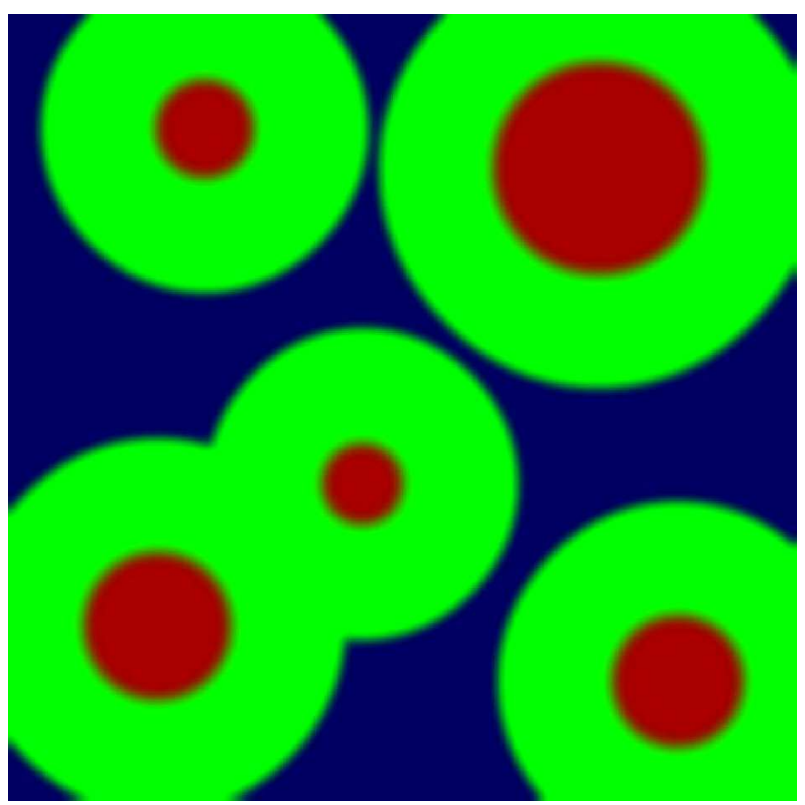

Рис. 6. Рост фазы BCC_A2 на границе между фазами Liquid и НCР_А3 для гафния при $T=2000 \mathrm{~K}$.

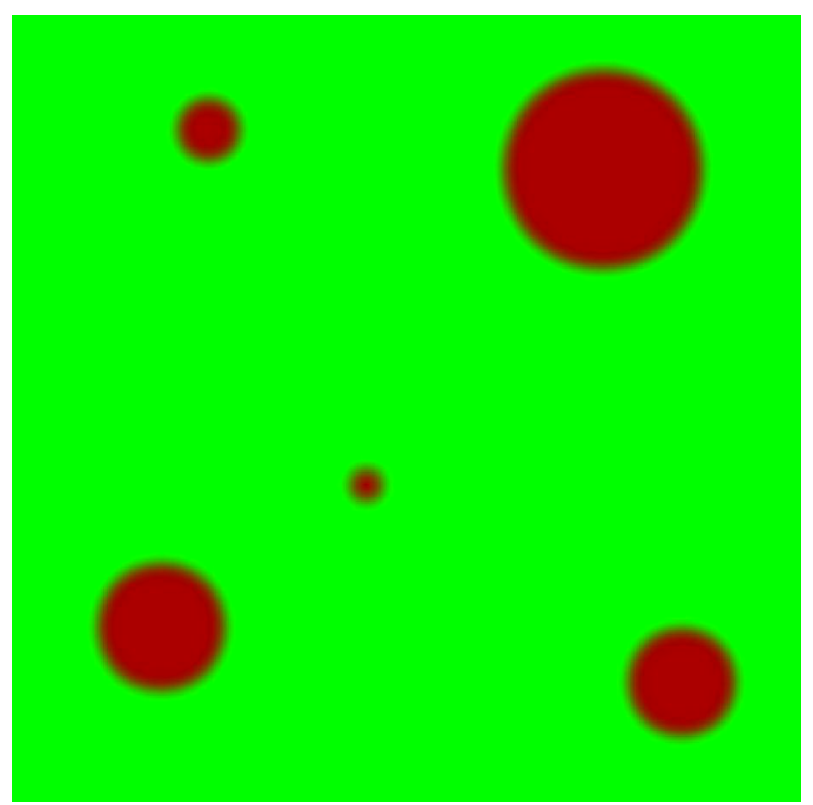

Рис. 7. Фаза ВCC_A2 заменила фазу Liquid и постепенно вытесняет фазу НCP_A3 для гафния при $T=2000 \mathrm{~K}$.

Liquid и НCР_A3 (рис. 5,6). Далее фаза ВCС_A2 замещает собой фазу Liquid и постепенно вытесняет фазу HCP_A3 (рис. 7).

\section{Заключение и выводы}

В работе представлен вывод многофазной локальнонеравновесной модели фазовых превращений в однокомпонентных системах. Преимуществом представленной 
модели является выполнение принципа соответствия с ранее известной двухфазной моделью фазового поля [26] и отсутствие необходимости явного отслеживания связи (1) при численных расчетах.

Выполнение принципа соответствия гарантирует правильное физическое поведение решений на межфазных границах и существование термодинамических стационарных состояний в системе, что отличает модель от ранее предложенных подходов.

Отсутствие необходимости явного отслеживания связи (1) при численном моделировании фазовых переходов в рамках данного подхода позволяет рассматривать уравнения всех фазовых полей как независимые. Последнее существенно упрощает технологию моделирования процессов структурообразования в многофазных системах, поскольку дает возможность решения уравнений фазового поля по отдельности. В ходе моделирования проверялось условие нормировки суммы фаз, которое выполнялось в любой момент времени расчета. Результаты моделирования совпадают с ожидаемым поведением неравновесной системы и подтверждают применимость представленной модели.

Предложенный подход может быть обобщен на случай растворов и является основой для дальнейшего развития метода фазового поля в науках о материалах.

\section{Финансирование работы}

Исследование выполнено при финансовой поддержке РФФИ и Правительства Удмуртской Республики в рамках научного проекта № 18-42-180002.

\section{Конфликт интересов}

Автор заявляет, что у него нет конфликта интересов.

\section{Список литературы}

[1] W.J. Sames, F.A. List, S. Pannala, R.R. Dehoff, S.S. Babu. Int. Mat. Rev., 61 (5), 315 (2016). DOI: $10.1080 / 09506608.2015 .1116649$

[2] N. Provatas, K. Elder. Phase-Field Methods in Materials Science and Engineering (Wiley-VCH Weinheim, 2010)

[3] A.A. Wheeler, W.J. Boettinger, G.B. McFadden. Phys. Rev. A, 45, 7424 (1992). DOI: 10.1103/PhysRevA.45.7424

[4] R. Kobayashi. Physica D, 63, 410 (1993). DOI: 10.1016/0167-2789(93)90120-P

[5] S.G. Kim, W.T. Kim, T. Suzuki. Phys. Rev. E, 60, 7186 (1999). DOI: 10.1103/PhysRevE.60.7186

[6] A. Karma, Y.H. Lee, M. Plapp. Phys. Rev. E, 61, 3996 (2000). DOI: 10.1016/S0167-2789(97)00226-1

[7] J.D. Van der Waals. J. Stat. Phys., 20 (2), 197 (1979).

[8] Л.Д. Ландау. ЖЭТФ, 7, 19 (1937). [L.D. Landau. JETP, 7, 19 (1937).]

[9] В.Л. Гинзбург, Л.Д. Ландау. ЖЭТФ, 20, 1064 (1950). [V.L. Ginsburg, L.D. Landau. JETP, 20, 1064 (1950).]

[10] J.W. Cahn, J.E. Hilliard. J. Chem. Phys., 28 (2), 258 (1958).

[11] S.M. Allen, J.W. Cahn. Acta Metall., 27, 1085 (1979).
[12] I. Steinbach, L. Zhang, M. Plapp. Acta Mater., 60, 2689 (2012). DOI: 10.1016/j.actamat.2012.01.035

[13] H.F. Wang, P.K. Galenko, X. Zhang, W.W. Kuang, F. Liu, D.M. Herlach. Acta Mater., 90, 982 (2015). DOI: $10.1016 /$ j.actamat.2015.02.021

[14] V.G. Lebedev, P.K. Galenko. Russ. Metall. (Metally), 8, 785 (2016). DOI: 10.1134/S0036029516080097

[15] J. Tiaden, B. Nestler, H.J. Diepers, I. Steinbach. Physica D, 115, 73 (1998). DOI: 10.1016/S0167-2789(97)00226-1

[16] I. Steinbach, F. Pezolla. Physica D, 134, 358 (1999). DOI: $10.1016 / \mathrm{S} 0167-2789(99) 00129-3$

[17] B. Nestler, A.A. Wheeler. Physica D, 138, 114 (2000). DOI: $10.1016 / \mathrm{S} 0010-4655(02) 00252-7$

[18] L.Q. Chen. Annu. Rev. Mater. Res., 32, 113 (2002). DOI: 10.1146/annurev.matsci.32.112001.132041

[19] B. Bottger, J. Eiken, M. Apel. Comp. Mat. Science, 108 (B), 283 (2015). DOI: 10.1016/J.COMMATSCI.2015.03.003

[20] B. Bottger, M. Apel, M. Budnitzki, J. Eiken, G. Laschet, B. Zhou. Comp. Mater. Science 184, 109909 (2020). DOI: 10.1016/j.commatsci.2020.109909

[21] B. Nestler, A.A. Wheeler. Phys. Rev. E, 57, 2602 (1998). DOI: 10.1103/PhysRevE.57.2602

[22] А.И. Лурье. Аналитическая механика (Физматлит, М., 1961)

[23] S.K. Vadlamani, T.P. Xiao, E. Yablonovitch. Proc. Nat. Acad. Sc., 117 (43), 26639 (2020). DOI: 10.1073/pnas.2015192117

[24] Ö. Yeniay, Math. Comp. Appl., 10 (1), 45 (2005). DOI: $10.3390 /$ mca10010045

[25] D. Kessler. J. Cryst. Growth, 224, 175 (2001). DOI: 10.1016/S0022-0248(01)00814-4

[26] В.Г. Лебедев, А.А. Лебедева, П.К. Галенко. Письма в ЖЭТФ, 101 (2), 143 (2015). [V.G. Lebedev, A.A. Lebedeva, P.K. Galenko. JETP Lett., 101 (2), 136 (2015). DOI: $10.1134 / \mathrm{S} 0021364015020101]$

[27] P. Galenko, D. Jou. Phys. Rev. E, 71, 046125 (2005). DOI: doi.org/10.1103/PhysRevE.71.046125

[28] A.T. Dinsdale. Calphad, 15 (4), 317 (1991). DOI: $10.1016 / 0364-5916(91) 90030-\mathrm{N}$

[29] А.А. Новокрещенова, В.Г. Лебедев. ЖТФ, 87 (4), 621 (2017). [A.A. Novokreshchenova, V.G. Lebedev. Tech. Phys., 62, 642 (2017). DOI: 10.1134/S1063784217040181] 